\title{
Adaptive Synchronization for Uncertain Delayed Fractional-Order Hopfield Neural Networks via Fractional-Order Sliding Mode Control
}

\author{
Bo Meng $\mathbb{D}^{1,2}$ and Xiaohong Wang ${ }^{2}$ \\ ${ }^{1}$ College of Electrical Engineering and Automation, Shandong University of Science and Technology, Qingdao 266590, China \\ ${ }^{2}$ College of Mathematics and Systems Science, Shandong University of Science and Technology, Qingdao 266590, China \\ Correspondence should be addressed to Bo Meng; mb0922@sdust.edu.cn
}

Received 17 May 2018; Accepted 10 July 2018; Published 18 July 2018

Academic Editor: Xue-Jun Xie

Copyright (C) 2018 Bo Meng and Xiaohong Wang. This is an open access article distributed under the Creative Commons Attribution License, which permits unrestricted use, distribution, and reproduction in any medium, provided the original work is properly cited.

Adaptive synchronization for a class of uncertain delayed fractional-order Hopfield neural networks (FOHNNs) with external disturbances is addressed in this paper. For the unknown parameters and external disturbances of the delayed FOHNNs, some adaptive estimations are designed. Firstly, a fractional-order switched sliding surface is proposed for the delayed FOHNNs. Then, according to the fractional-order extension of the Lyapunov stability criterion, a fractional-order sliding mode controller is constructed to guarantee that the synchronization error of the two uncertain delayed FOHNNs converges to an arbitrary small region of the origin. Finally, a numerical example of two-dimensional uncertain delayed FOHNNs is given to verify the effectiveness of the proposed method.

\section{Introduction}

The research of neural networks (NNs) is quite extensive, reflecting the characteristics of multidisciplinary technology. NNs have many successful applications in the fields of associative memories and image processing. Recently, the discussion on NNs has become a hot topic [1-3]. Guo et al. [4] studied the exponential stability analysis for complex-valued memristor-based bidirectional associative memory (BAM) NNs with time delays. Lv et al. [5] used NNs to discuss the adaptive tracking control for a class of uncertain nonlinear systems. Li et al. [6] studied Hopf bifurcation analysis of complex-valued neural networks model.

Fractional calculus (FC) has a long history. As early as 1695 , the concept of fractional differential was mentioned in Leibnitz's letter to L'Hospital. For a long time, FC continues to grow. Podlubny's book [7] systematically introduced the concepts and properties of FC. Bai et al. (see [8-13], and the references therein) studied the existence and uniqueness of solutions for fractional differential equations (FDE). Wang et al. [14-16] studied the numerical analysis of FDE. In recent years, fractional-order systems (FOS) have attracted wide attentions. The control problems of all kinds of FOS were studied recently [17-21]. Many researchers focused on fractional-order neural networks (FONNs) [22-27]. Cao et al. [28] investigated the existence and uniqueness of the nontrivial solution of NNs and the uniform stability of the FONNs.

The researches on the stability of NNs, FOS, and stochastic systems have attracted the attention of a large number of researchers, and many achievements have been made [29-41]. The sliding mode control (SMC) is a very popular strategy for a general class of nonlinear uncertain systems, with a very large frame of applications fields. Due to the use of the discontinuous function, its main features are the robustness of closed-loop system and the finite-time convergence. Utkin et al. [42] studied the minimum possible value of control based on adaptation SMC methodology. Efe. et al. [43] discussed the fractional fuzzy adaptive SMC. Aghababa [44] designed a chatter-free terminal sliding mode controller for nonlinear fractional-order dynamical systems. The synchronization problems of FOHNNs have captured more and more researchers' attention [45-48]. Xi et al. [22] have discussed SMC for uncertain FOHNNs. Liu et al. 
[24] have researched adaptive synchronization of a class of FONNs. It is well known that time delay is unavoidable due to finite switching speeds of the amplifiers, and it may cause oscillations or instability of dynamic systems. Wang et al. [26] have discussed the stability analysis of FOHNNs with time delay.

However, to the best of our knowledge, there are few attentions to adaptive synchronization for a class of uncertain delayed FOHNNs subject to external disturbances. The SMC technology was used to solve the above problems in the paper. The rest of this paper is organized as follows: some necessary definitions and lemmas are given in Section 2. The main works including the introduction of fractional-order network model, the fractional-order switched sliding mode surface (SMS), the design of adaptive synchronization controller, and stability analysis are included in Section 3. Section 4 presents a simulation example. Finally, the paper is concluded in Section 5.

\section{Preliminaries}

There are several kinds of definitions for fractional-order derivatives. The definitions of more frequency of use in literatures are Grünwald-Letnikov, Riemann-Liouville, and Caputo definitions [7]. These definitions are generally not equivalent with each other. The Caputo's derivative's Laplace transform requires integer-order derivatives for the initial conditions, which was used in engineering applications frequently. But, the Riemann-Liouville definition's Laplace transform involved fractional-order derivatives for the initial conditions. It was hard to use physically. In the following parts, the Caputo's derivative will be used [24]. Firstly, we give some definitions and lemmas.

Definition 1 (Riemann-Liouville fractional-order integral [7]). The Riemann-Liouville fractional integral of order $\alpha$ for a function $f(t)$ is defined as

$$
{ }_{t_{0}} D_{t}^{-\alpha} f(t)=\frac{1}{\Gamma(\alpha)} \int_{t_{0}}^{t} \frac{f(\tau)}{(t-\tau)^{1-\alpha}} d \tau
$$

where $\alpha>0, t \geq t_{0} . \Gamma(\alpha)$ is Euler's gamma function.

The gamma function $\Gamma(\alpha)$ is defined for all complex numbers except the nonpositive integers. For complex numbers with a positive real part, it is defined via a convergent infinite integral:

$$
\Gamma(\alpha)=\int_{0}^{+\infty} \tau^{\alpha-1} e^{-\tau} d \tau
$$

Definition 2 (Caputo fractional-order derivative [7]). The Caputo fractional derivative of order $\alpha$ for a function $f(t)$ is defined as

$$
{ }_{t_{0}}^{C} D_{t}^{\alpha} f(t)=\frac{1}{\Gamma(n-\alpha)} \int_{t_{0}}^{t}(t-\tau)^{n-\alpha-1} f^{(n)}(\tau) d \tau .
$$

where $\alpha>0, n$ is an integer satisfying $n-1 \leq \alpha<n$. Particularly, for $0<\alpha<1$ case, one can get

$$
{ }_{t_{0}}^{C} D_{t}^{\alpha} f(t)=\frac{1}{\Gamma(1-\alpha)} \int_{t_{0}}^{t}(t-\tau)^{-\alpha} f^{\prime}(\tau) d \tau .
$$

According to Definition 2, for any constants $L_{1} \in \mathbf{R}$ and $L_{2} \in \mathbf{R}$, the linearity of Caputo's fractional derivative is described by

$$
{ }_{t_{0}}^{C} D_{t}^{\alpha}\left(L_{1} f(t)+L_{2} g(t)\right)=L_{1 t_{0}}^{C} D_{t}^{\alpha} f(t)+L_{2 t_{0}}^{C} D_{t}^{\alpha} g(t) .
$$

In nonlinear control systems, Lyapunov second method gives a way to analyze the stability of the system without explicitly solving the differential equations. The Lyapunov stability theory for FOS has been developed by Li et al. [33]. One of the main contributions of [33] is the following lemma.

Consider the fractional-order nonlinear system:

$$
\begin{gathered}
{ }_{t_{0}}^{C} D_{t}^{\alpha} x(t)=f(x, t), \\
x\left(t_{0}\right)=x_{t_{0}},
\end{gathered}
$$

where $x(t) \in \mathbf{R}^{n}$ is the state vector and $f(x, t) \in \mathbf{R}^{n}$ is a Lipschitz continuous nonlinear function.

Lemma 3 (see [49]). Let $G(t)$ be a continuous function on $[0,+\infty)$, if there exist constants $\kappa_{1}>0$ and $\kappa_{2}>0$, such that

$$
{ }_{t_{0}}^{C} D_{t}^{\alpha} G(t) \leq-\kappa_{1} G(t)+\kappa_{2}, \quad t \geq 0
$$

Then,

$$
G(t) \leq G(0) E_{\alpha}\left(-\kappa_{1} t^{\alpha}\right)+\kappa_{2} t^{\alpha} E_{\alpha, \alpha+1}\left(-\kappa_{1} t^{\alpha}\right), \quad t \geq 0 .
$$

where $0<\alpha<1, E_{\alpha}(\cdot)$ and $E_{\alpha, \alpha+1}(\cdot)$ are one-parameter Mittag-Leffler function and two-parameter Mittag-Leffler function, respectively.

Remark 4. Mittag-Leffler stability means asymptotical stability [32].

Lemma 5 (see [7]). If $x(t) \in C^{1}[0, T]$, for $\alpha>0$ and $T>0$, then the following equations hold:

$$
{ }_{0}^{C} D_{t}^{\alpha}\left({ }_{0} D_{t}^{-\alpha} x(t)\right)=x(t) .
$$

and

$$
{ }_{0} D_{t}^{-\alpha}\left({ }_{0}^{C} D_{t}^{\alpha} x(t)\right)=x(t)-\sum_{k=0}^{n-1} \frac{x^{(k)}(t)}{k !} t^{k} .
$$

In particular, for $0<\alpha<1$,

$$
{ }_{0} D_{t}^{-\alpha}\left({ }_{0}^{C} D_{t}^{\alpha} x(t)\right)=x(t)-x(0) .
$$

Lemma 6 (see [21]). Let $x(t) \subset \mathbf{R}^{n}$ be a continuous and derivable function. Then for any $t>0$ the following inequality holds:

$$
\frac{1}{2}{ }_{0}^{C} D_{t}^{\alpha} x^{T}(t) x(t) \leq x^{T}(t)_{0}^{C} D_{t}^{\alpha} x(t)
$$




\section{Main Results}

In this section, considering a system of the uncertain FOHNNs with delay (as a master system)

$$
\begin{aligned}
{ }_{0}^{C} D_{t}^{\alpha} x_{i}(t)= & -a_{i}(t) x_{i}(t)+\sum_{j=1}^{n} b_{i j} f_{j}\left(x_{j}(t)\right) \\
& +\sum_{j=1}^{n} c_{i j} g_{j}\left(x_{j}(t-\tau)\right)+I_{i} .
\end{aligned}
$$

where $i=1,2, \ldots, n, 0<\alpha<1, n$ is the number of units in a neural network, $x_{i}(t)$ is the state of the $i$ th unit at time $t, f_{j}, g_{j}$ denotes the activation function of the $j$ th neuron, $b_{i j}, c_{i j}$ denotes the constant connection weight of the $j$ th neuron on the $i$ th neuron, $a_{i}>0$ represents the rate with which the $i$ th neuron resets its potential to the resting state when disconnected from the network and $a_{i}>0$ is unknown, $I_{i}$ denotes the constant external inputs, and $\tau$ is the transmission constant delay.

Let us discuss the synchronization results, assuming that (13) is a master system and the slave system is defined by the following equation:

$$
\begin{aligned}
{ }_{0}^{C} D_{t}^{\alpha} y_{i}(t)= & -a_{i}(t) y_{i}(t)+\sum_{j=1}^{n} b_{i j} f_{j}\left(y_{j}(t)\right) \\
& +\sum_{j=1}^{n} c_{i j} g_{j}\left(y_{j}(t-\tau)\right)+I_{i}+d_{i}(t) \\
& +u_{i}(t) .
\end{aligned}
$$

where $y_{i}(t)$ is the state of the $i$ th unit at time $t, d_{i}(t)$ is the unknown external disturbance, and $u_{i}(t)$ is the control input which will be given later.

Defining the synchronization error $e_{i}(t)$ as

$$
e_{i}(t)=y_{i}(t)-x_{i}(t)
$$

then the error dynamics between the master system (13) and the slave system (14) can be written as

$$
\begin{aligned}
{ }_{0}^{C} D_{t}^{\alpha} e_{i}(t)= & -a_{i}(t) e_{i}(t) \\
& +\sum_{j=1}^{n} b_{i j}\left(f_{j}\left(y_{j}(t)\right)-f_{j}\left(x_{j}(t)\right)\right) \\
& +\sum_{j=1}^{n} c_{i j}\left(g_{j}\left(y_{j}(t-\tau)\right)-g_{j}\left(x_{j}(t-\tau)\right)\right) \\
& +d_{i}(t)+u_{i}(t) .
\end{aligned}
$$

Assumption 7 . Assuming that the nonlinear functions $f_{j}$ and $g_{j}(j=1,2, \ldots, n)$ satisfy local Lipschitz conditions, and existing positive constants $L_{j}^{1}$ and $L_{j}^{2}$ such that

$$
\begin{aligned}
& \left|f_{j}\left(y_{j}(t)\right)-f_{j}\left(x_{j}(t)\right)\right| \leq L_{j}^{1}\left|y_{j}(t)-x_{j}(t)\right|, \\
& \left|g_{j}\left(y_{j}(t-\tau)\right)-g_{j}\left(x_{j}(t-\tau)\right)\right| \\
& \quad \leq L_{j}^{2}\left|y_{j}(t-\tau)-x_{j}(t-\tau)\right| .
\end{aligned}
$$

Assumption 8. Let the external disturbance $d_{i}(t)(i=1,2$, $\ldots, n)$ be a bounded continuous function, so there exists an unknown positive constant $\rho_{i}$ such that

$$
\left|d_{i}(t)\right| \leq \rho_{i}
$$

For the sake of simplicity, this article only discusses the constant unknown disturbance.

3.1. Controller Design. Generally, designing the process of SMC has two steps. Firstly, an appropriate SMS is designed, which represents the required system dynamic characteristics. In this paper, a switching fractional-order SMS is given as

$$
s_{i}(t)={ }_{0} D_{t}^{-1}\left({ }_{0}^{C} D_{t}^{\alpha} e_{i}(t)+p_{i} e_{i}(t)+q_{i} \operatorname{sign}\left(e_{i}(t)\right)\right),
$$

where $i=1,2, \ldots, n, e_{i}(t)$ is the state of the error system (16), and $p_{i}$ and $q_{i}$ are positive constants. sign( $(\cdot)$ is the symbolic function.

$$
\operatorname{sign}\left(e_{i}(t)\right)= \begin{cases}1 & e_{i}(t)>0, \\ -1 & e_{i}(t)<0, \\ \in[-1,1] & e_{i}(t)=0 .\end{cases}
$$

According to the SMC theory, when the system operates in SMS, the SMS and its derivative must satisfy

$$
\begin{gathered}
s_{i}(t)=0, \\
\dot{s}_{i}(t)=0 .
\end{gathered}
$$

As a result, considering ((19)-(21)), one obtains

$$
\dot{s}_{i}(t)={ }_{0}^{C} D_{t}^{\alpha} e_{i}(t)+p_{i} e_{i}(t)+q_{i} \operatorname{sign}\left(e_{i}(t)\right)=0 .
$$

Then, we have the sliding mode equation (SME)

$$
{ }_{0}^{C} D_{t}^{\alpha} e_{i}(t)=-\left(p_{i} e_{i}(t)+q_{i} \operatorname{sign}\left(e_{i}(t)\right)\right) .
$$

In the next parts, we construct the SMC law $u_{i}(t)$ to make sure the state trajectories of system (16) reach the SMS $s_{i}(t)=0$ and keep on it forever by the SMC method. The fractionalorder SMC law is presented as

$$
\begin{gathered}
u_{i}(t)=-\left(-\widehat{a}_{i}(t)+p_{i}\right) e_{i}(t)-\widehat{d}_{i}(t)-\left|s_{i}(t)\right| \\
\cdot\left(\sum_{j=1}^{n}\left|b_{i j}\right| L_{j}^{f}\left|e_{j}(t)\right|+\sum_{j=1}^{n}\left|c_{i j}\right| L_{j}^{g}\left|e_{j}(t-\tau)\right|\right) \\
-q_{i} \operatorname{sign}\left(e_{i}(t)\right)-\zeta_{i}^{a} s_{i}(t)-\zeta_{i}^{b} \operatorname{sign}\left(s_{i}(t)\right),
\end{gathered}
$$

where $\zeta_{i}^{a}>0$ and $\zeta_{i}^{b}>0$ are constant gains, $\widehat{a}_{i}(t)$ is the estimation of $a_{i}(t), \hat{d}_{i}(t)$ is the estimation of $d_{i}(t)$, and the unknown parameters $a_{i}(t)$ and $d_{i}(t)$ are estimated as

$$
\begin{aligned}
& \dot{\vec{a}}_{i}(t)=-\eta_{i}^{a} s_{i}(t) e_{i}(t), \\
& \dot{\hat{d}}_{i}(t)=\eta_{i}^{d} s_{i}(t) .
\end{aligned}
$$

where $i=1,2, \ldots, n, \eta_{i}^{a}>0$ and $\eta_{i}^{d}>0$ are adaptation gains. 
In order to realize SMC, two steps are required. Firstly, the system trajectories are controlled to reach the SMS $s_{i}(t)=0$, which is shown in Theorem 9. Secondly, once the system operates in SMS, we should get the stability of the error system (16) and make sure SMS converge to zero in finite time, which is shown in Theorem 12.

Theorem 9. For the uncertain delayed FOHNNs (16), if the system is controlled by the SMC law (24) and (25), then the system trajectories will converge to the $S M S s_{i}(t)=0$ in finite time.

Proof. Choose the positive definite Lyapunov function candidate

$$
\begin{aligned}
V_{i}(t)= & \frac{1}{2} s_{i}(t)^{2}+\frac{1}{2 \eta_{i}^{a}}\left(\widehat{a}_{i}(t)-a_{i}(t)\right)^{2} \\
& +\frac{1}{2 \eta_{i}^{d}}\left(\widehat{d}_{i}(t)-d_{i}\right)^{2} .
\end{aligned}
$$

Taking the integer-order derivative of $V_{i}(t)$, we have

$$
\begin{aligned}
\dot{V}_{i}(t)= & s_{i}(t) \dot{s}_{i}(t)+\frac{1}{\eta_{i}^{a}}\left(\widehat{a}_{i}(t)-a_{i}\right) \dot{\vec{a}}_{i}(t) \\
& +\frac{1}{\eta_{i}^{d}}\left(\widehat{d}_{i}(t)-d_{i}\right) \dot{\hat{d}}_{i}(t) .
\end{aligned}
$$

Inserting $\dot{s}_{i}(t)$ from (22) into the above equation, one has

$$
\begin{aligned}
\dot{V}_{i}(t)= & s_{i}(t)\left({ }_{0}^{C} D_{t}^{\alpha} e_{i}+p_{i} e_{i}+q_{i} \operatorname{sign}\left(e_{i}\right)\right) \\
& +\frac{1}{\eta_{i}^{a}}\left(\widehat{a}_{i}(t)-a_{i}\right) \dot{\vec{a}}_{i}(t) \\
& +\frac{1}{\eta_{i}^{d}}\left(\hat{d}_{i}(t)-d_{i}\right) \dot{\hat{d}}_{i}(t) .
\end{aligned}
$$

Based on (16), we get

$$
\begin{aligned}
& \dot{V}_{i}(t)=s_{i}(t)\left(-a_{i} e_{i}(t)\right. \\
& +\sum_{j=1}^{n} b_{i j}\left(f_{j}\left(y_{j}(t)\right)-f_{j}\left(x_{j}(t)\right)\right) \\
& +\sum_{j=1}^{n} c_{i j}\left(g_{j}\left(y_{j}(t-\tau)\right)-g_{j}\left(x_{j}(t-\tau)\right)\right)+d_{i} \\
& \left.+u_{i}(t)+p_{i} e_{i}(t)+q_{i} \operatorname{sign}\left(e_{i}(t)\right)\right)+\frac{1}{\eta_{i}^{a}}\left(\widehat{a}_{i}(t)\right. \\
& \left.\quad-a_{i}\right) \dot{\hat{a}}_{i}(t)+\frac{1}{\eta_{i}^{d}}\left(\widehat{d}_{i}(t)-d_{i}(t)\right) \dot{\vec{d}}_{i}(t) .
\end{aligned}
$$

According to Assumption 7, we will obtain

$$
\begin{aligned}
& s_{i}(t) \sum_{j=1}^{n} b_{i j}\left(f_{j}\left(y_{j}(t)\right)-f_{j}\left(x_{j}(t)\right)\right) \\
& \leq\left|s_{i}(t)\right| \sum_{j=1}^{n}\left|b_{i j}\right|\left|f_{j}\left(y_{j}(t)\right)-f_{j}\left(x_{j}(t)\right)\right| \\
& \leq\left|s_{i}(t)\right| \sum_{j=1}^{n}\left|b_{i j}\right| L_{j}^{f}\left|e_{j}(t)\right|
\end{aligned}
$$

Correspondingly, we have

$$
\begin{aligned}
& s_{i}(t) \sum_{j=1}^{n} c_{i j}\left(g_{j}\left(y_{j}(t-\tau)\right)-g_{j}\left(x_{j}(t-\tau)\right)\right) \\
& \leq\left|s_{i}(t)\right| \sum_{j=1}^{n}\left|c_{i j}\right| L_{j}^{g}\left|e_{j}(t-\tau)\right|
\end{aligned}
$$

Combining ((29)-(31)), we can get the following conclusion:

$$
\begin{aligned}
& \dot{V}_{i}(t) \\
& \leq s_{i}(t)\left(\left(-a_{i}+p_{i}\right) e_{i}(t)+q_{i} \operatorname{sign}\left(e_{i}(t)\right)+d_{i}+u_{i}(t)\right) \\
& \quad+\left|s_{i}(t)\right| \sum_{j=1}^{n}\left|b_{i j}\right| L_{j}^{f}\left|e_{j}(t)\right| \\
& \quad+\left|s_{i}(t)\right| \sum_{j=1}^{n}\left|c_{i j}\right| L_{j}^{g}\left|e_{j}(t-\tau)\right| \\
& \quad+\frac{1}{\eta_{i}^{a}}\left(\widehat{a}_{i}(t)-a_{i}\right) \dot{\hat{a}}_{i}(t)+\frac{1}{\eta_{i}^{d}}\left(\widehat{d}_{i}(t)-d_{i}\right) \dot{\vec{d}}_{i}(t) .
\end{aligned}
$$

Substituting $u_{i}(t)$ from (24) into (32), it yields

$$
\begin{gathered}
\dot{V}_{i}(t) \leq s_{i}(t)\left(\left(-a_{i}+p_{i}\right) e_{i}(t)+q_{i} \operatorname{sign}\left(e_{i}(t)\right)+d_{i}\right. \\
-\left(-\widehat{a}_{i}+p_{i}\right) e_{i}(t)-\widehat{d}_{i}(t)-\left|s_{i}(t)\right| \\
\cdot\left(\sum_{j=1}^{n}\left|b_{i j}\right| L_{j}^{f}\left|e_{j}(t)\right|+\sum_{j=1}^{n}\left|c_{i j}\right| L_{j}^{g}\left|e_{j}(t-\tau)\right|\right) \\
-q_{i} \operatorname{sign}\left(e_{i}(t)\right)-\zeta_{i}^{a} s_{i}(t)-\zeta_{i}^{b} \operatorname{sign}\left(s_{i}(t)\right)+\left|s_{i}(t)\right| \\
\left.\cdot \sum_{j=1}^{n}\left|b_{i j}\right| L_{j}^{f}\left|e_{j}(t)\right|+\left|s_{i}(t)\right| \sum_{j=1}^{n}\left|c_{i j}\right| L_{j}^{g}\left|e_{j}(t-\tau)\right|\right) \\
+\frac{1}{\eta_{i}^{a}}\left(\widehat{a}_{i}(t)-a_{i}\right) \dot{\hat{a}}_{i}(t)+\frac{1}{\eta_{i}^{d}}\left(\widehat{d}_{i}(t)-d_{i}\right) \dot{\vec{d}}_{i}(t) .
\end{gathered}
$$


Through operation, we get

$$
\begin{aligned}
& \dot{V}_{i}(t) \leq s_{i}(t)\left(\left(\widehat{a}_{i}(t)-a_{i}\right) e_{i}(t)-\left(\widehat{d}_{i}(t)-d_{i}\right)\right. \\
& \left.\quad-\zeta_{i}^{a} s_{i}(t)-\zeta_{i}^{b} \operatorname{sign}\left(s_{i}(t)\right)\right)+\frac{1}{\eta_{i}^{a}}\left(\widehat{a}_{i}(t)-a_{i}\right) \dot{\hat{a}}_{i}(t) \\
& \quad+\frac{1}{\eta_{i}^{d}}\left(\widehat{d}_{i}(t)-d_{i}\right) \dot{\hat{d}}_{i}(t) .
\end{aligned}
$$

Insert (25)

$$
\begin{aligned}
& \dot{V}_{i}(t) \leq s_{i}(t)\left(\left(\widehat{a}_{i}(t)-a_{i}\right) e_{i}(t)-\left(\widehat{d}_{i}(t)-d_{i}\right)\right. \\
& \left.\quad-\zeta_{i}^{a} s_{i}(t)-\zeta_{i}^{b} \operatorname{sign}\left(s_{i}(t)\right)\right)-\frac{1}{\eta_{i}^{a}}\left(\widehat{a}_{i}(t)-a_{i}\right) \eta_{i}^{a} s_{i}(t) \\
& \quad \cdot e_{i}(t)+\frac{1}{\eta_{i}^{d}}\left(\widehat{d}_{i}(t)-d_{i}\right) \eta_{i}^{d} s_{i}(t)
\end{aligned}
$$

Then, one obtains

$$
\dot{V}_{i}(t) \leq-\zeta_{i}^{a} s_{i}^{2}(t)-\zeta_{i}^{b} \operatorname{sign}\left(s_{i}(t)\right) s_{i}(t) .
$$

Using $\operatorname{sign}\left(s_{i}(t)\right) s_{i}(t)=\left|s_{i}(t)\right|$ and property of inequality, we get

$$
\dot{V}_{i}(t) \leq-\zeta_{i}^{b}\left|s_{i}(t)\right|<0
$$

where $\zeta_{i}^{b}>0$. Therefore, according to Lyapunov theory, the system states will converge to SMS $s_{i}(t)=0$. Hence, the proof is achieved completely.

Remark 10. Theorem 9 gets the error systems trajectories to reach the sliding surface $s_{i}(t)=0$ in finite time.

Remark 11. Time delay and external disturbance have little influence on the error system (16).

3.2. Stability of Sliding Mode. For the SME (23), we choose the positive definite Lyapunov function

$$
V_{i}(t)=\frac{1}{2} e_{i}^{2}(t) .
$$

Taking the fractional-order derivative of $V_{i}(t)$ and using Lemma 6, we get

$$
\begin{aligned}
{ }_{0}^{C} D_{t}^{\alpha} V_{i}(t) & \leq e_{i}(t){ }_{0}^{C} D_{t}^{\alpha} e_{i}(t) \\
& =e_{i}(t)\left(-p_{i} e_{i}-q_{i} \operatorname{sign}\left(e_{i}(t)\right)\right) \\
& =-p_{i} e_{i}^{2}(t)-q_{i} e_{i}(t) \operatorname{sign}\left(e_{i}(t)\right) \\
& =-p_{i} e_{i}^{2}(t)-q_{i}\left|e_{i}(t)\right| \leq-q_{i}\left|e_{i}(t)\right| \\
& =-\sqrt{2} q_{i} V_{i}^{1 / 2}(t),
\end{aligned}
$$

where $q_{i}>0$. As a result, according to Lemma 3 and Remark $4, e_{i}$ will converge to 0 asymptotically.

Therefore, the state trajectories of system (23) will converge to 0 , so one has the following conclusion.
Theorem 12. The sliding mode dynamics system (23) is asymptotically stable, and its states $e_{i}(t)$ converge to 0 .

Corollary 13. By Theorems 9 and 12, system (16) is asymptotically stable, which means that system (14) can synchronize system (13).

\section{Numerical Simulations}

The effectiveness of the obtained theoretical results is shown by the example. Considering the two-dimensional uncertain delayed FOHNNs (as the Master system)

$$
\begin{aligned}
{ }_{0}^{C} D_{t}^{\alpha} x_{1}(t)= & -x_{1}+0.5 \sin \left(x_{1}(t)\right)+\sin \left(x_{2}(t)\right) \\
& +0.5 \tanh \left(x_{1}(t-0.8)\right) \\
& +\tanh \left(x_{2}(t-0.8)\right)+0.2, \\
{ }_{0}^{C} D_{t}^{\alpha} x_{2}(t)= & -0.5 x_{2}+\sin \left(x_{1}(t)\right)-0.5 \sin \left(x_{2}(t)\right) \\
& -0.5 \tanh \left(x_{1}(t-0.8)\right) \\
& -\tanh \left(x_{2}(t-0.8)\right)+0.3
\end{aligned}
$$

where $\alpha=0.9$, the initial conditions are $x_{1}(0)=-5, x_{2}(0)=$ 5.

The form of the slave system is given by

$$
\begin{aligned}
{ }_{0}^{C} D_{t}^{\alpha} y_{1}(t)= & -y_{1}+0.5 \sin \left(y_{1}(t)\right) \\
& +\sin \left(y_{2}(t)\right) 0.5 \tanh \left(y_{1}(t-0.8)\right) \\
& +\tanh \left(y_{2}(t-0.8)\right)+0.2+0.1 \\
& +u_{1}(t), \\
{ }_{0}^{C} D_{t}^{\alpha} y_{2}(t)= & -0.5 y_{2}-\sin \left(y_{1}(t)\right)-0.5 \sin \left(y_{2}(t)\right) \\
& -0.5 \tanh \left(y_{1}(t-0.8)\right) \\
& -\tanh \left(y_{2}(t-0.8)\right)+0.3+0.15 \\
& +u_{2}(t)
\end{aligned}
$$

Assume that the initial conditions are $y_{1}(0)=-3, y_{2}(0)=3$, and $\alpha=0.9$.

Choosing $p_{1}=p_{2}=0.5, q_{1}=q_{2}=1.5, L_{1}^{f}=L_{2}^{f}=L_{1}^{g}=$ $L_{1}^{g}=1, \eta_{1}^{a}=\eta_{2}^{a}=1.1, \eta_{1}^{d}=\eta_{2}^{d}=1.2, \zeta_{1}^{a}=\zeta_{2}^{a}=1.1, \zeta_{1}^{b}=\zeta_{2}^{b}=$ 1.2 , one gets

$$
\begin{aligned}
& \dot{\hat{a}}_{1}(t)=-1.1 e_{1}(t) s_{1}(t) \\
& \dot{\hat{a}}_{2}(t)=-1.1 e_{2}(t) s_{2}(t)
\end{aligned}
$$

and

$$
\begin{aligned}
& \dot{\hat{d}}_{1}(t)=1.2 s_{1}(t) \\
& \dot{\hat{d}}_{2}(t)=1.2 s_{2}(t)
\end{aligned}
$$




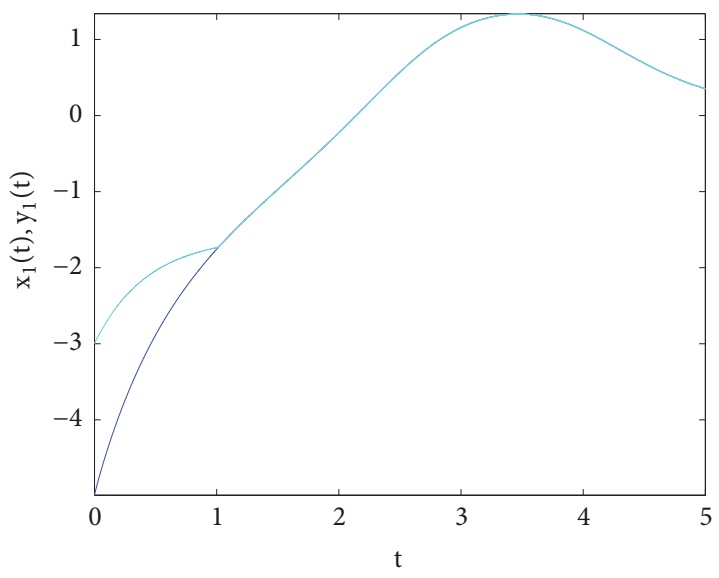

FIGURE 1: Synchronization between $x_{1}(t)$ (blue line) and $y_{1}(t)$ (green line).

We use (19) and design the SMS

$$
\begin{aligned}
& s_{1}(t) \\
& \quad={ }_{0} D_{t}^{-1}\left({ }_{0}^{C} D_{t}^{0.9} e_{1}(t)+0.5 e_{1}(t)+1.5 \operatorname{sign}\left(e_{1}(t)\right)\right) \\
& s_{2}(t) \\
& \quad={ }_{0} D_{t}^{-1}\left({ }_{0}^{C} D_{t}^{0.9} e_{2}(t)+0.5 e_{2}(t)+1.5 \operatorname{sign}\left(e_{2}(t)\right)\right) .
\end{aligned}
$$

Thus, according to (24), the control inputs are obtained as

$$
\begin{gathered}
u_{1}(t)=\left(\widehat{a}_{1}(t)-0.5\right) e_{1}(t)-\widehat{d}_{1}(t)-1.5 \operatorname{sign}\left(e_{1}(t)\right) \\
\quad-1.1 s_{1}(t)-1.2 \operatorname{sign}\left(s_{1}(t)\right)-\left|s_{1}(t)\right|\left(0.5\left|e_{1}(t)\right|\right. \\
\left.+\left|e_{2}(t)\right|+0.5\left|e_{1}(t-0.8)\right|+\left|e_{2}(t-0.8)\right|\right), \\
u_{2}(t)=\left(\widehat{a}_{2}(t)-0.5\right) e_{2}(t)-\widehat{d}_{2}(t)-1.5 \operatorname{sign}\left(e_{2}(t)\right) \\
\quad-1.1 s_{2}(t)-1.2 \operatorname{sign}\left(s_{2}(t)\right)-\left|s_{2}(t)\right|\left(-\left|e_{1}(t)\right|\right. \\
\left.-0.5\left|e_{2}(t)\right|-0.5\left|e_{1}(t-0.8)\right|-1\left|e_{2}(t-0.8)\right|\right) .
\end{gathered}
$$

The simulation results are depicted in Figures 1-6. Figures 1-3 show the synchronization between two fractional-order neural networks and the time response of the synchronization errors. The time response of the updated parameters and the sliding surfaces are included in Figures 4, 5, and 6, respectively. From the results, we can see that the synchronization errors converge to origin rapidly, and favorable synchronization performance has been achieved.

\section{Conclusion}

The adaptive synchronization problem for FOHNNs with system uncertainties, time delay, and external disturbances has been studied by SMC. Some estimations for system uncertainties and external disturbances are made. Firstly, establishing a switched SMS, the finite-time stability of the SMS to origin is proved according to the fractional-order

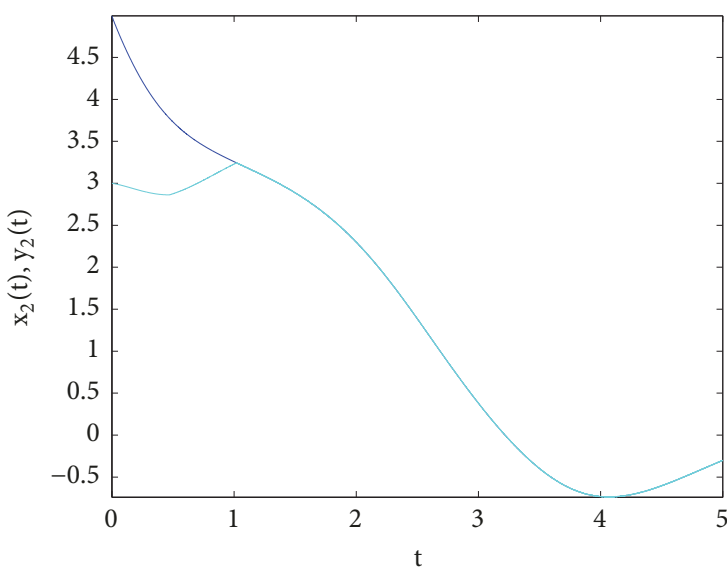

FIgURE 2: Synchronization between $x_{2}(t)$ (blue line) and $y_{2}(t)$ (green line).

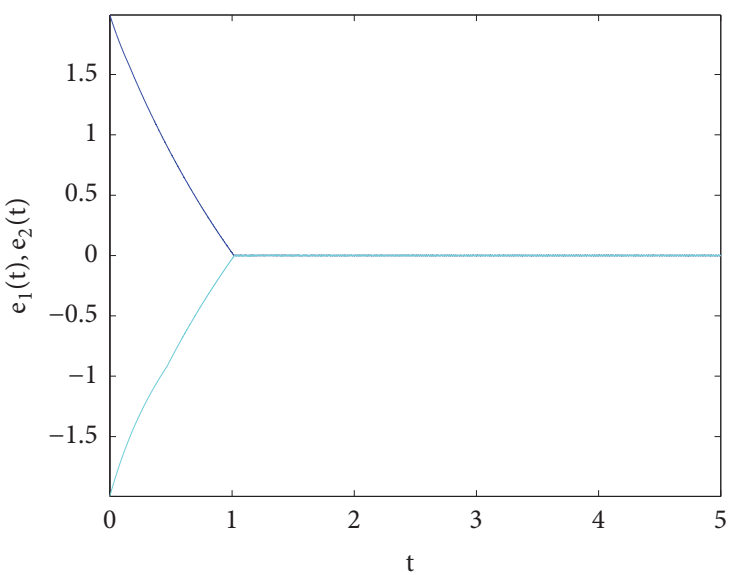

FIGURE 3: Synchronization errors $e_{1}(t)$ (blue line), $e_{2}(t)$ (green line).

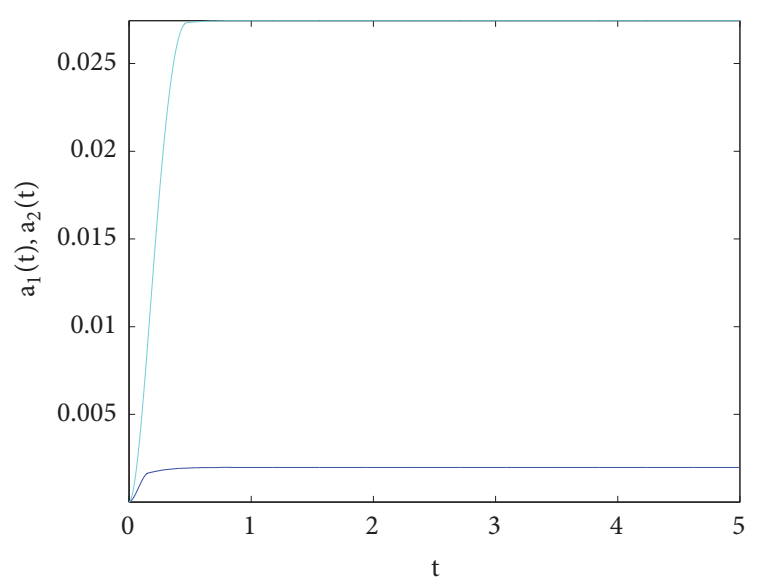

FIGURE 4: The time response of control parameters: $\widehat{a}_{1}$ (blue line) and $\hat{a}_{2}$ (green line).

Lyapunov theory. Secondly, an adaptive synchronization fractional-order sliding mode controller is designed to force the error systems trajectories to reach the switching SMS and remain on it forever. The effectiveness and feasibility of theoretical results are verified by the numerical simulations. 


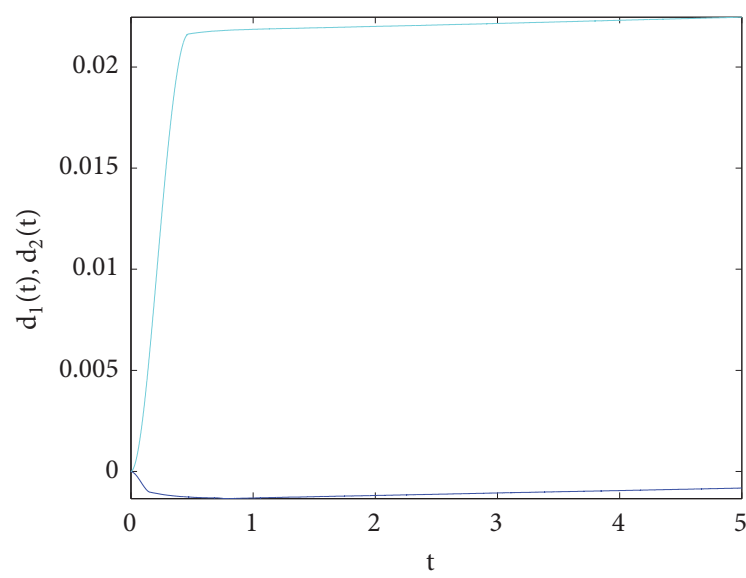

FIGURE 5: The time response of control parameters: $\widehat{d}_{1}$ (blue line) and $\widehat{d}_{2}$ (green line).

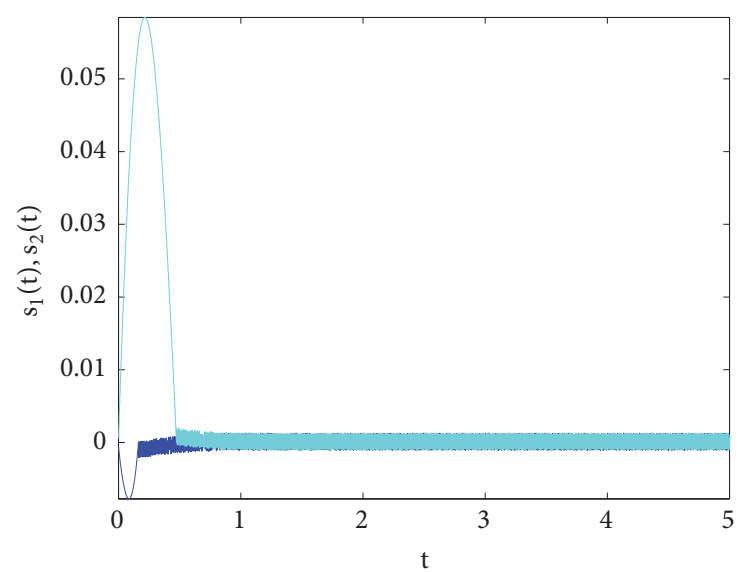

Figure 6: The sliding surfaces: $s_{1}(t)$ (blue line) and $s_{2}(t)$ (green line).

Furthermore, taking advantage of the SMC theory, the stability problems of FOHNNs with both multiple time delays and impulses will be discussed in future works.

\section{Data Availability}

No data were used to support this study.

\section{Conflicts of Interest}

The authors declare that there are no conflicts of interest regarding the publication of this article.

\section{Acknowledgments}

This work was supported by the National Natural Science Foundation of China (no. 61573008), Post-Doctoral Applied Research Projects of Qingdao (no. 2015122), and Scientific Research Foundation of Shandong University of Science and Technology for Recruited Talents (no. 2014RCJJ032).

\section{References}

[1] G. Velmurugan, R. Rakkiyappan, V. Vembarasan, J. Cao, and A. Alsaedi, "Dissipativity and stability analysis of fractionalorder complex-valued neural networks with time delay," Neural Networks, vol. 86, pp. 42-53, 2017.

[2] I. Aizenberg, "Complex-valued neural networks with multivalued neurons," Springer, pp. 39-62, 2011.

[3] R. Rakkiyappan, G. Velmurugan, and J. Cao, "Multiple $\mu$ stability analysis of complex-valued neural networks with unbounded time-varying delays," Neurocomputing, vol. 149, pp. 594-607, 2015.

[4] R. Guo, Z. Zhang, X. Liu, and C. Lin, "Existence, uniqueness, and exponential stability analysis for complex-valued memristor-based BAM neural networks with time delays," Applied Mathematics and Computation, vol. 311, pp. 100-117, 2017.

[5] W. Lv and F. Wang, "Adaptive tracking control for a class of uncertain nonlinear systems with infinite number of actuator failures using neural networks," Advances in Difference Equations, Paper No. 374, 16 pages, 2017.

[6] L. Li, Z. Wang, Y. Li, H. Shen, and J. Lu, "Hopf bifurcation analysis of a complex-valued neural network model with discrete and distributed delays," Applied Mathematics and Computation, vol. 330, pp. 152-169, 2018.

[7] I. Podlubny, "Fractional differential equations," Mathematics in Science and Engineering, 1998.

[8] Y. Cui, "Uniqueness of solution for boundary value problems for fractional differential equations," Applied Mathematics Letters, vol. 51, pp. 48-54, 2016.

[9] Z. Bai, X. Dong, and C. Yin, "Existence results for impulsive nonlinear fractional differential equation with mixed boundary conditions," Boundary Value Problems, vol. 1, pp. 63-71, 2016.

[10] Z. Bai, Y. Chen, H. Lian, and S. Sun, "On the existence of blow up solutions for a class of fractional differential equations," Fractional Calculus and Applied Analysis, vol. 17, no. 4, pp. 11751187, 2014.

[11] R. Pu, X. Zhang, Y. Cui, P. Li, and W. Wang, "Positive solutions for singular semipositone fractional differential equation subject to multipoint boundary conditions," Journal of Function Spaces, vol. 2017, pp. 1-7, 2017.

[12] Y. Zou and G. He, "On the uniqueness of solutions for a class of fractional differential equations," Applied Mathematics Letters, vol. 74, pp. 68-73, 2017.

[13] Y. Cu, W. Ma, Q. Sun, and X. Su, "New uniqueness results for boundary value problem of fractional differential equation," Nonlinear Analysis: Modelling and Control, pp. 31-39, 2018.

[14] J. Sabatier, O. P. Agrawal, and A. J. T. Machado, Advances in fractional calculus, Springer Netherlands, 2007.

[15] Z. Wang, "A numerical method for delayed fractional-order differential equations," Journal of Applied Mathematics, vol. 2013, Article ID 256071, 7 pages, 2013.

[16] Z. Wang, X. Huang, and J. Zhou, "A numerical method for delayed fractional-order differential equations: based on G-L definition," Applied Mathematics \& Information Sciences, vol. 7, no. 2, pp. 525-529, 2013.

[17] B. Xin and Y. Li, "0-1 test for chaos in a fractional order financial system with investment incentive," Abstract and Applied Analysis, Art. ID 876298, 10 pages, 2013.

[18] Z. Wang, X. Wang, Y. Li, and X. Huang, "Stability and Hopf bifurcation of fractional-order complex-valued single neuron 
model with time delay," International Journal of Bifurcation and Chaos, vol. 27, no. 13, 1750209, 13 pages, 2017.

[19] Z. Wang, X. Huang, and G. D. Shi, "Analysis of nonlinear dynamics and chaos in a fractional order financial system with time delay," Computers \& Mathematics with Applications, vol. 62, no. 3, pp. 1531-1539, 2011.

[20] I. Petras, "Modeling and numerical analysis of fractional-order bloch equations," Computers \& Mathematics with Applications, vol. 61, no. 2, pp. 341-356, 2011.

[21] N. Aguila-Camacho, M. A. Duarte-Mermoud, and J. A. Gallegos, "Lyapunov functions for fractional order systems," Communications in Nonlinear Science and Numerical Simulation, vol. 19, no. 9, pp. 2951-2957, 2014.

[22] Y. Xi, Y. Yu, S. Zhang, and X. Hai, "Finite-time robust control of uncertain fractional-order Hopfield neural networks via sliding mode control," Chinese Physics B, vol. 27, no. 1, 010202, 2018.

[23] L. Chen, Y. Chai, R. Wu, T. Ma, and H. Zhai, "Dynamic analysis of a class of fractional-order neural networks with delay," Neurocomputing, vol. 111, pp. 190-194, 2013.

[24] H. Liu, S. Li, H. Wang, Y. Huo, and J. Luo, "Adaptive synchronization for a class of uncertain fractional-order neural networks," Entropy, vol. 17, no. 10, pp. 7185-7200, 2015.

[25] G. A. Anastassiou, "Fractional neural network approximation," Computers \& Mathematics with Applications, vol. 64, no. 6, pp. 1655-1676, 2012.

[26] H. Wang, Y. Yu, G. Wen, and S. Zhang, "Stability analysis of fractional-order neural networks with time delay," Neural Processing Letters, vol. 42, no. 2, pp. 479-500, 2015.

[27] Y. Fan, X. Huang, Z. Wang, and Y. Li, "Nonlinear dynamics and chaos in a simplified memristor-based fractional-order neural network with discontinuous memductance function," Nonlinear Dynamics, vol. 93, no. 2, pp. 611-627, 2018.

[28] C. Song and J. Cao, "Dynamics in fractional-order neural networks," Neurocomputing, vol. 142, pp. 494-498, 2014.

[29] J. Zhou, C. Sang, X. Li, M. Fang, and Z. Wang, "Ho consensus for nonlinear stochastic multi-agent systems with time delay," Applied Mathematics and Computation, vol. 325, pp. 41-58, 2018.

[30] J. Wang, K. Liang, X. Huang, Z. Wang, and H. Shen, "Dissipative fault-tolerant control for nonlinear singular perturbed systems with Markov jumping parameters based on slow state feedback," Applied Mathematics and Computation, vol. 328, pp. 247-262, 2018.

[31] H. Gao, J. Xia, G. Zhuang, Z. Wang, and Q. Sun, "Nonfragile finite-time extended dissipative control for a class of uncertain switched neutral systems," Complexity, vol. 2017, pp. 1-22, 2017.

[32] Y. Li, Y. Chen, and I. Podlubny, "Stability of fractional-order nonlinear dynamic systems: Lyapunov direct method and generalized Mittag-Leffler stability," Computers \& Mathematics with Applications, vol. 59, no. 5, pp. 1810-1821, 2010.

[33] Y. Li, Y. Chen, and I. Podlubny, "Mittag-Leffler stability of fractional order nonlinear dynamic systems," Automatica, vol. 45, no. 8, pp. 1965-1969, 2009.

[34] H. Ma and Y. Jia, "Stability analysis for stochastic differential equations with infinite Markovian switchings," Journal of Mathematical Analysis and Applications, vol. 435, no. 1, pp. 593-605, 2016.

[35] Z. Yan and W. Zhang, "Finite-time stability and stabilization of Itô-type stochastic singular systems," Abstract and Applied Analysis, vol. 2014, pp. 1-10, 2014.

[36] Y. Li, W. Zhang, and X. Liu, "Stability of nonlinear stochastic discrete-time systems," Journal of Applied Mathematics, vol. 2013, Article ID 356746, 8 pages, 2013.
[37] Y. Li, Y. Sun, and F. Meng, "New criteria for exponential stability of switched time-varying systems with delays and nonlinear disturbances," Nonlinear Analysis: Hybrid Systems, vol. 26, pp. 284-291, 2017.

[38] Y. Lin and W. Zhang, "Necessary/sufficient conditions for Pareto optimum in cooperative difference game," Optimal Control Applications and Methods, vol. 39, no. 2, pp. 1043-1060, 2018.

[39] Y. Lin, T. Zhang, and W. Zhang, "Pareto-based guaranteed cost control of the uncertain mean-field stochastic systems in infinite horizon," Automatica, vol. 92, pp. 197-209, 2018.

[40] L. Yao and W. Zhang, "Adaptive tracking control for a class of random pure-feedback nonlinear systems with Markovian switching," International Journal of Robust and Nonlinear Control, vol. 28, no. 8, pp. 3112-3126, 2018.

[41] W. Zhang, Y. Lin, and L. Xue, "Linear quadratic Pareto optimal control problem of stochastic singular systems," Journal of The Franklin Institute, vol. 354, no. 2, pp. 1220-1238, 2017.

[42] V. I. Utkin and A. S. Poznyak, "Adaptive sliding mode control, advances in sliding mode control," Springer Berlin Heidelberg, pp. 21-53, 2013.

[43] M. Ö. Efe, "Fractional fuzzy adaptive sliding-mode control of a 2-DOF direct-drive robot arm," IEEE Transactions on Systems Man and Cybernetics Part B Cybernetics A Publication of the IEEE Systems Man and Cybernetics Society, vol. 38, no. 6, pp. 1561-1570, 2008.

[44] M. P. Aghababa, "Design of a chatter-free terminal sliding mode controller for nonlinear fractional-order dynamical systems," International Journal of Control, vol. 86, no. 10, pp. 1744-1756, 2013.

[45] K. Liang, M. Dai, H. Shen, J. Wang, Z. Wang, and B. Chen, "L2-Lo synchronization for singularly perturbed complex networks with semi-Markov jump topology," Applied Mathematics and Computation, vol. 321, pp. 450-462, 2018.

[46] Z. Zhang, H. Shao, Z. Wang, and H. Shen, "Reduced-order observer design for the synchronization of the generalized Lorenz chaotic systems," Applied Mathematics and Computation, vol. 218, no. 14, pp. 7614-7621, 2012.

[47] X. Huang, Y. Fan, J. Jia, Z. Wang, and Y. Li, "Quasisynchronisation of fractional-order memristor-based neural networks with parameter mismatches," IET Control Theory \& Applications, vol. 11, no. 14, pp. 2317-2327, 2017.

[48] J. Chen, Z. Zeng, and P. Jiang, "Global Mittag-Leffler stability and synchronization of memristor-based fractional-order neural networks," Neural Networks, vol. 51, pp. 1-8, 2014.

[49] A. Wu and Z. Zeng, "Global Mittag-Leffler stabilization of fractional-order memristive neural networks," IEEE Transactions on Neural Networks and Learning Systems, vol. 28, no. 1, pp. 206-217, 2017. 


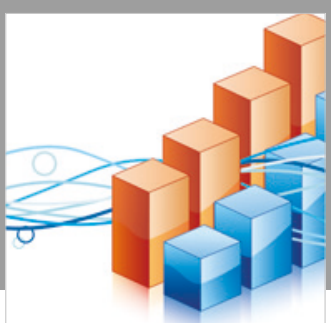

Advances in

Operations Research

\section{-n-m}
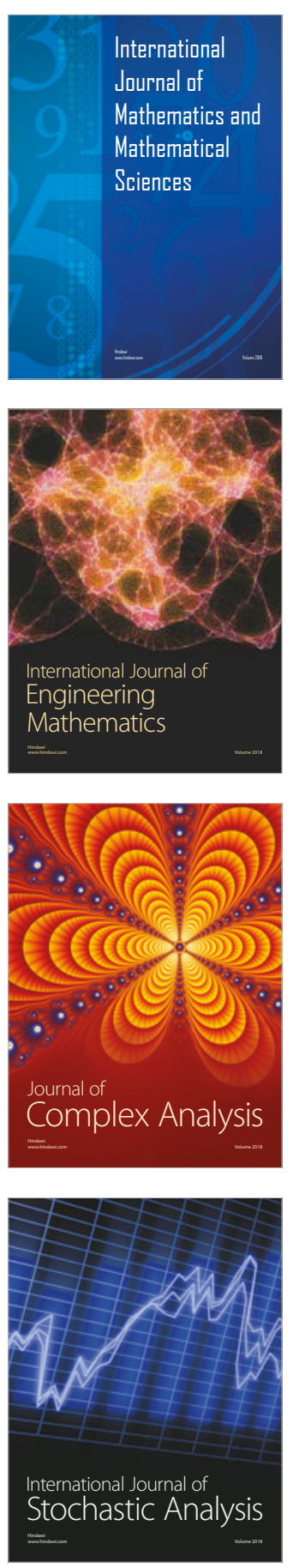
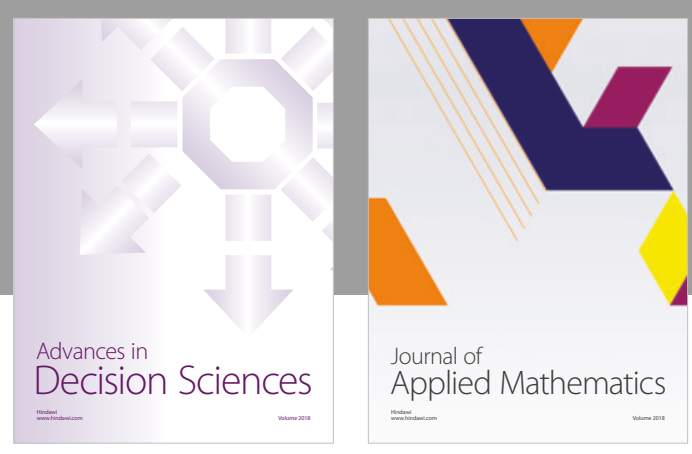

Journal of

Applied Mathematics
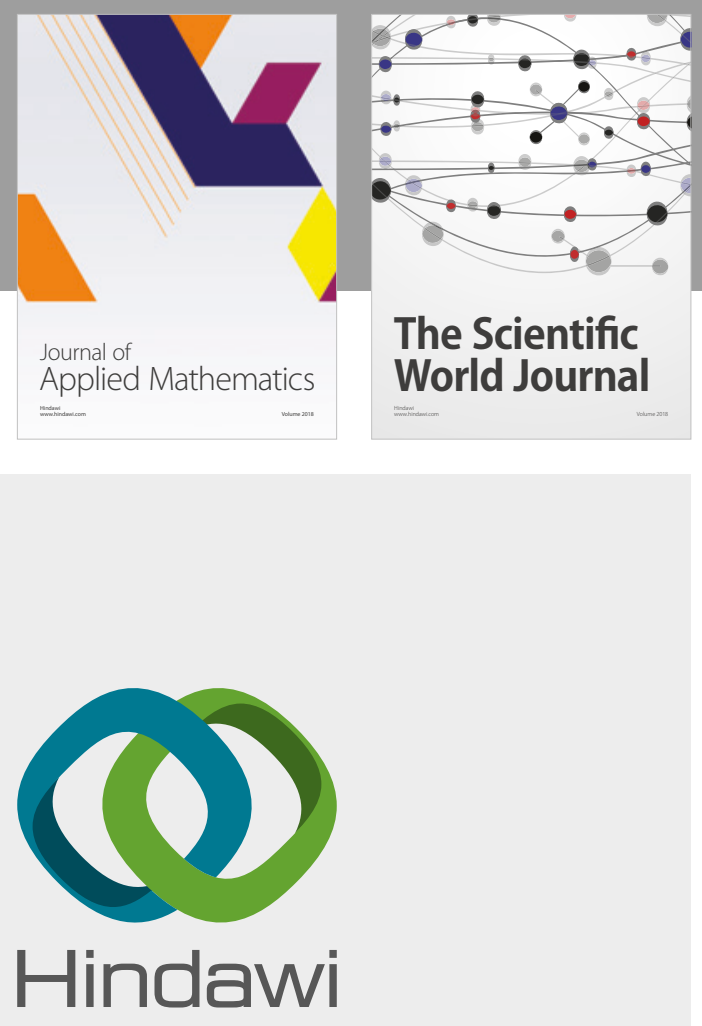

Submit your manuscripts at

www.hindawi.com

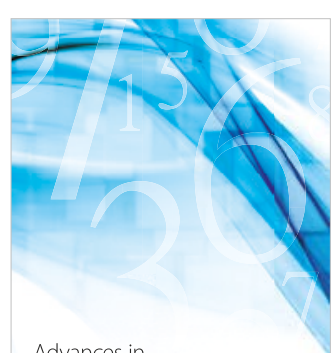

Advances in
Numerical Analysis
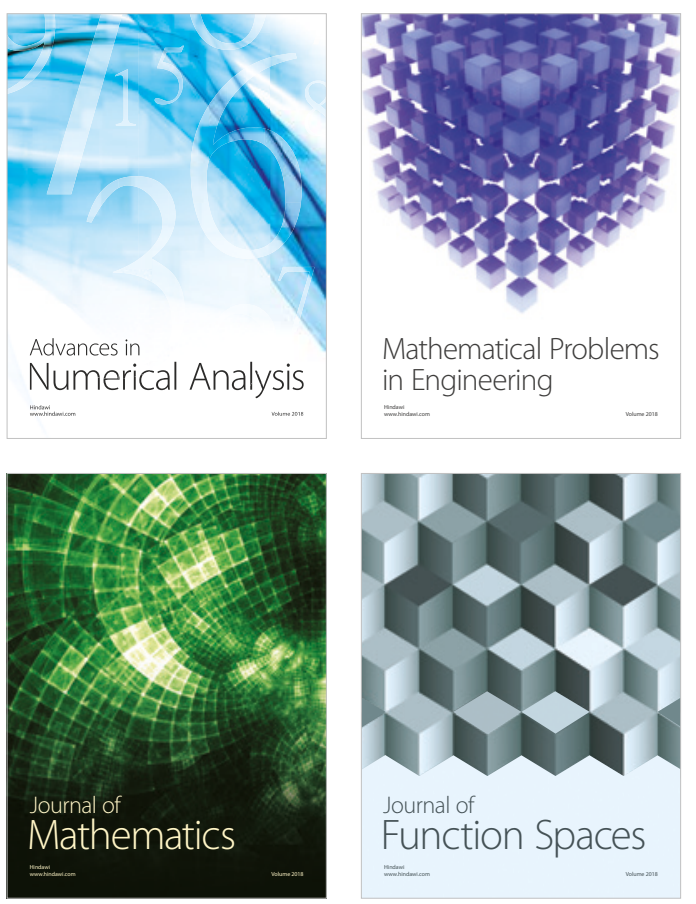

Mathematical Problems in Engineering

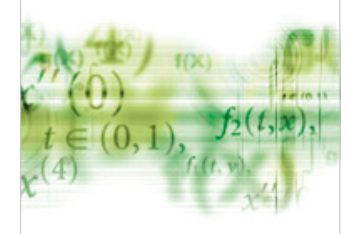

International Journal of

Differential Equations

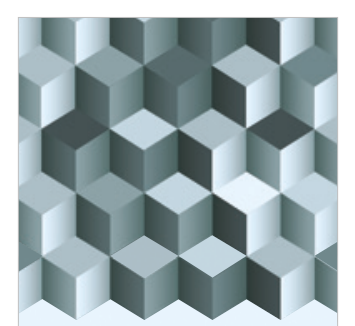

Journal of

Function Spaces

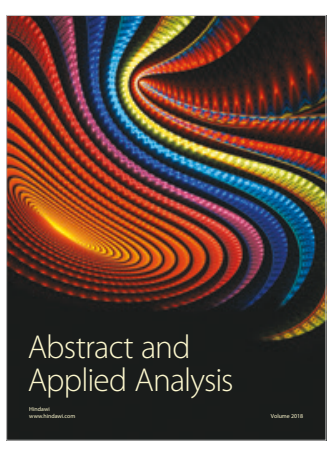

The Scientific

World Journal

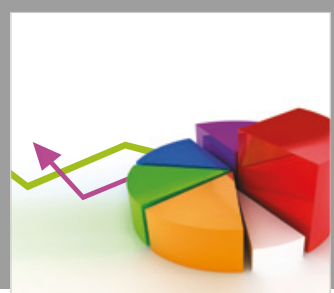

Journal of

Probability and Statistics
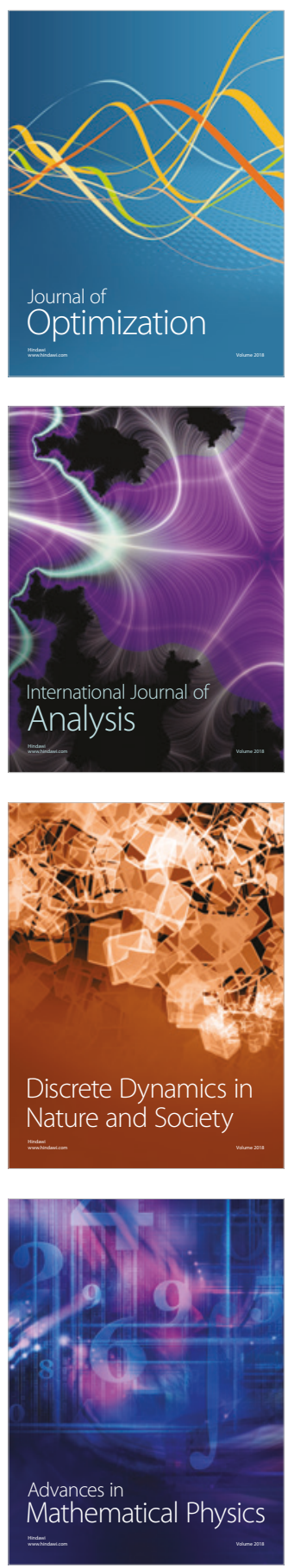